\title{
Whole blood cell parameters help us predict the prognosis in patients with acute ST segment elevation myocardial infarction: Myth or reality?
}

We have read the recently published article by Çiçek et al. "White blood cell count to mean platelet volume ratio: A novel and promising prognostic marker for ST-segment elevation myocardial infarction" with great interest [1]. In that well-designed article, the authors aimed to evaluate the predictive value of admission white blood cell count (WBC) to mean platelet volume (MPV) ratio (WMR) on prognosis in 2,603 consecutive patients with ST elevation myocardial infarction (STEMI), undergoing primary percutaneous coronary intervention (pPCI). They found that WMR has the highest area under receiver operating characteristic (ROC) curve, and pairwise comparisons of the ROC curves revealed that WMR has higher discriminative ability for long-term mortality than WBC, MPV, red blood cell distribution with (RDW), WBC-MPV combination, and platelet to lymphocyte ratio and neutrophil to lymphocyte ratio (PLR-NLR) combination in patients undergoing $\mathrm{pPCI}$ for STEMI. They concluded that higher WMR value on admission was associated with worse outcomes in patients with STEMI and independently predicted the long-term mortality better than other complete blood count components, such as MPV, RDW, and PLR-NLR and WBC-MPV combinations.

The 'no-reflow' phenomenon in patients with STEMI is associated with worse prognosis at short- and long-term follow-up periods [2, 3]. The mechanisms responsible for the 'no-reflow' phenomenon have not been clearly understood yet. Pathophysiology of 'no-reflow' phenomenon comprises reperfusion injury, distal atherothrombotic embolization, ischemic injury, and increased susceptibility of coronary microcirculation to injury [2].

Automated blood cell counters are commonly available in clinical laboratories and they can be used to determine RDW, platecrit, platelet count, platelet distribution width (PDW), MPV and some ratios, e.g. neutrophil-lymphocyte ratio (NLR),
RDW-platelet ratio (RPR), PLR-NLR and WBC-MPV ratios. It was shown that some hematologic parameters such as MPV, RDW, NLR and PLR may play a role in predicting worse outcomes in patients with STEMI undergoing pPCI [4-6]. Our group previously found that admission PDW and MPV are independent correlates of 'no-reflow' and in-hospital major adverse cardiac events (MACEs) among patients with STEMI undergoing pPCI [7]. Moreover, we also showed that female gender, pain to balloon time, high Thrombolysis in Myocardial Infarction (TIMI) thrombus grade, tirofiban, MPV, and PLR were independent predictors of 'no-reflow' in young patients with STEMI after pPCI [8]. What is more, we tried to evaluate the relations between pre-procedural RPR and the in-hospital and long-term outcomes in 580 STEMI patients undergoing pPCI [9]. In that study, pain to balloon time, multivessel disease, TIMI thrombus grade, tirofiban, aspirin, previous coronary artery disease, NLR, platecrit, and RPR remained independent predictors of 'no-reflow' after pPCI on multivariate analysis. Patients in 'no-reflow' group tended to constitute a higher percentage of in-hospital MACE, including nonfatal myocardial infarction and cardiovascular mortality compared to the reflow patients. We concluded that admission NLR, platecrit, and RPR are independent correlates of 'no-reflow' and in-hospital MACEs among patients with STEMI undergoing pPCI.

In summary, admission complete blood cell parameters are easily obtained bedside for the prediction of short- and long-term mortality in patients with STEMI undergoing pPCI. From that point of view we strongly believe that further large-scale prospective trials are urgently needed to clarify the exact role of complete blood cell parameters in predicting short- and long-term mortalities in that patient setting.

\section{Conflict of interest: None declared}




\section{References}

1. Çiçek G, Açıkgöz SK, Yayla Ç, Kundi H, İleri M. White blood cell count to mean platelet volume ratio: A novel and promising prognostic marker for ST-segment elevation myocardial infarction. Cardiol J, 2016; 23: 225-235. doi: 10.5603/CJ.a2016.0001.

2. Wong DTL, Puri R, Richardson JD, Worthley MI, Worthley SG. Myocardial "no-reflow": Diagnosis, pathophysiology and treatment. Int J Cardiol, 2013; 167: 1798-806. doi: 10.1016/j.ijcard.2012.12.049.

3. Topsakal R, Kaya MG, Karakaya E et al. Relationship between no-reflow phenomenon and serotonin levels in patients with acute ST-elevation myocardial infarction who underwent primary percutaneous intervention. Anadolu Kardiyol Derg, 2010; 10: 253-259.

4. Çiçek G, Açıkgoz SK, Bozbay M et al. Neutrophil-lymphocyte ratio and platelet-lymphocyte ratio combination can predict prognosis in patients with ST-segment elevation myocardial infarction undergoing primary percutaneous coronary intervention. Angiology, 2015; 66: 441-447. doi: 10.1177/00033197145 35970 .
5. Acet H, Ertaş F, Akıl MA et al. Novel predictors of infarct-related artery patency for ST-segment elevation myocardial infarction: Platelet-to-lymphocyte ratio, uric acid, and neutrophil-to-lymphocyte ratio. Anatol J Cardiol, 2015; 15: 648-656. doi: 10.5152/akd.2014.5592.

6. Uyarel H, Ergelen M, Cicek G et al. Red cell distribution width as a novel prognostic marker in patients undergoing primary angioplasty for acute myocardial infarction. Coron Artery Dis, 2011; 22: 138-144. doi: 10.1097/MCA.0b013e328342c77b.

7. Celik T, Kaya MG, Akpek M et al. Predictive value of admission platelet volume indices for in-hospital major adverse cardiovascular events in acute ST-segment elevation myocardial infarction. Angiology, 2015; 66: 155-62. doi: 10.1177/0003319713513493.

8. Celik T, Balta S, Ozturk C et al. Predictors of no-reflow phenomenon in young patients with acute ST-segment elevation myocardial infarction undergoing primary percutaneous coronary intervention. Angiology, 2015. doi: 10.1177/0003319715605977.

9. Celık T, Balta S, Demır M et al. Predictive value of admission red cell distribution width-platelet ratio for no-reflow phenomenon in acute ST segment elevation myocardial infarction undergoing primary percutaneous coronary intervention. Cardiol J, 2015; doi: 10.5603/CJ.a2015.0070.

Turgay Celık, Sevket Balta, Cengiz Ozturk, Atila Iyısoy

Department of Cardiology, School of Medicine, Gulhane Military Medical Academy, 06018 Etlik-Ankara, Turkey, tel: +90-312-3044268, fax: +90-312-3044250, e-mail: benturgay@yahoo.com 